\title{
Bubbles without toil or trouble
}

A surprisingly simple technique permits the rapid generation of submicrometersized vesicles from mammalian cells; these vesicles recreate the membrane and cytoplasmic environment of the parent cell, potentially enabling a broad range of nanoscale cellular screens.

Thinking small isn't always a bad thing. The immense high-throughput studies that are all the rage these days would be thoroughly unwieldy if innovative scientists didn't keep coming up with ways to work at an ever-tinier scale. A new contribution to this 'downsizing' comes from Horst Vogel and his colleagues at the Swiss Federal Institute of Technology (Lausanne, Switzerland), with the recent publication of a method for generating tiny vesicles that can partially replicate the functional environment of the mammalian cells from which they were derived (Pick et al., 2005).

The technique is simplicity itself. Cells are treated with the drug cytochalasin B, which rapidly disrupts the actin cytoskeleton; as the plasma membrane becomes unanchored, long tubular extensions form from the treated cell, and with gentle agitation, these can be sheared off to form tiny, surprisingly monodisperse vesicles. By varying the drug dosage or the extent of agitation, vesicle size can be modulated to produce particles ranging from a few hundred nanometers to a few micrometers in diameter.

Unlike with earlier techniques, the resulting vesicles seem to capture the cellular environment at the time of disruption. Says Vogel, "The nice thing for biophysical, biochemical and bioanalytical applications is that... you have in the vesicles the plasma membrane with all receptors retained in the same orientation as in the original cell, as well as parts of the cytoplasm comprising components of the cell signaling machinery."

Vogel's team confirmed this by generating vesicles from HEK293 cells expressing the serotonin $5-\mathrm{HT}_{3}$ receptor. Binding assays with fluorescently tagged and radiolabeled ligands confirmed that the receptor was presented in its proper orientation and that it had normal binding kinetics. Follow-up studies with a receptor agonist showed that $5-\mathrm{HT}_{3} \mathrm{R}$ binding triggered a transient uptake of calcium ions by the vesicle that was followed by a gradual restoration of the initial calcium concentration, demonstrating that the receptor maintained normal function and that the vesicle membranes also retained appropriate proteins for calcium ion export. These findings demonstrate the potential for conducting nanoscale signaling studies in such vesicles.

This technique could prove valuable for studies in which access to the appropriate cell type is a limiting factor. "When you just work with the cell, you can typically do one experiment," Vogel explains, "[But] when you prepare from a single cell between 50 and 100 vesicles, you can do 50 to 100 different experiments where you can control and manipulate the vesicles individually." In an earlier paper, Vogel's group demonstrated the ability to arrange vesicles of this scale onto arrays for highthroughput studies (Stamou et al., 2003), a method that should prove particularly useful in conjunction with this vesicle harvesting strategy.

His group is now working on several refinements to this system, including the development of a microfluidics-based approach for the rapid generation and sorting of vesicles. With enough high-quality vesicles, the potential for conducting the sort of high-throughput studies that were once restricted to whole-cell assays is nearly limitless: along with other signaling-related projects, Vogel describes ongoing projects to develop arrays of vesicles containing different $\mathrm{G}$ protein-coupled receptors. "We want to do bioanalytics on the smallest scale," he says. "When you scale down complex cellular reactions to 100 or 200 nanometers, and it's still functioning in some respects like a biological cell, that is the final unit where you can study signal transduction, and we want to do that for biophysics and biochemical screening." Michael Eisenstein

\section{RESEARCH PAPERS}

Pick, H. et al. Investigating cellular signaling reactions in single attoliter vesicles. J. Am. Chem Soc. 127, 2908-2912 (2005)

Stamou, D. et al. Self-assembled microarrays of attoliter molecular vessels. Angew. Chem. Int. Ed. Engl. 42, 5580-5583 (2003). 